\title{
Expression and clinical significance of PD- L1 and BRAF expression in nasopharyngeal carcinoma
}

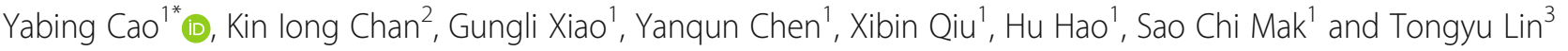

\begin{abstract}
Background: The prognostic value of programmed death-ligand 1 (PD-L1) and BRAF expression in nasopharyngeal carcinoma (NPC) is not well-defined. In this study we investigated alterations in PD-L1, BRAF and EGFR by using immunohistochemistry analysis in a cohort of consecutively enrolled NPC patients.

Methods: A retrospective review of 154 NPC patients form our previous study (BMC Cancer. 2013; 13:226) were conducted. Survival and prognostic impacts were analyzed based on PD-L1, BRAF and EGFR expression levels.

Results: One hundred fifty four patients were included in this study. PD-L1 expression was detected in $87.7 \%$ of patients; $14.3 \%$ had 1-5\% PD-L1 expression, $47.4 \%$ had 5-49\% expression while $26 \%$ had $\geq 50 \%$ expression Higher PD-L1 expression was significantly associated with shorter PFS and OS. The median PFS was 25 months (95\% Cl 15.7-34.3 months) and OS was 35 months (95\% Cl 22.60-47.4 months) for patients with PD-L1 expression 250\%; both median PFS and OS were not yet reached for patients with PD-L1 expression < 50\%. PFS was significantly higher in BRAF mutation positive patients (5-year PFS: $55.1 \%$ vs. 30.8\%, $P=0.044$ ).
\end{abstract}

Conclusion: Tumor PD-L1 expression and BRAF mutation are associated with poor outcomes in patients with NPC. This study was retrospectively registered in ClinicalTrials.gov (NCT03989297) on 2019-6-18.

Keywords: Nasopharyngeal carcinoma, Programmed death-ligand 1, BRAF, Prognosis

\section{Background}

Nasopharyngeal carcinoma (NPC) is rare in most parts of the world but is one of the more common types of cancer in southern China. In 2015, it was estimated that the incidence of NPC was 60.6 per 100,000 in China with a mortality rate of 34.1 per 100,000 [1,2]. The main treatment for NPC is radiotherapy or chemoradiotherapy [3], and the 5-year survival rate is about $85 \%$ [4]. Even with best available treatment, about $30 \%$ of patients relapse with local recurrence or metastasis [5]. The prognosis for patients with recurrent or primary metastatic NPC is poor with a median progression free survival of 19.4 months [6]. Evidently, novel approaches and better therapies are needed for the treatment of NPC.

Biomarkers that can reliably predict the prognosis of patients are important. In a previous study, we found

\footnotetext{
* Correspondence: sumscaoyabing@hotmail.com

'Department of Oncology, Kiang Wu Hospital, Macau, SAR, China Full list of author information is available at the end of the article
}

that gender and age were strong independent prognostic factors for NPC [7]. Specifically, younger and male patients were more likely to have distant metastases and exhibit poorer overall survival and progression-free survival rates compared to other NPC patients treated in our center [7]. A more recent study identified a prognostic gene expression-based signature that predicts distant metastasis in locoregionally advanced NPC [8].

In addition to prognostic biomarkers, predictive biomarkers that can identify patients who are likely to benefit from a particular therapy can help guide treatment selection. NPC is characterized by lymphocyte infiltration, including T cells and cytotoxic tumor-infiltrating $\mathrm{T}$ lymphocytes [9]. Since immune checkpoint inhibitors can activate cytotoxic $\mathrm{T}$ cells to attack cancer cells, patients with lymphocyte-rich cancer types (such as EBV-positive NPC) may benefit more from immunotherapy $[10,11]$. Tumor programmed deathligand 1 (PD-L1) expression levels have also been suggested to be of predictive value for treatment efficacy in some

(c) The Author(s). 2019 Open Access This article is distributed under the terms of the Creative Commons Attribution 4.0 International License (http://creativecommons.org/licenses/by/4.0/), which permits unrestricted use, distribution, and reproduction in any medium, provided you give appropriate credit to the original author(s) and the source, provide a link to the Creative Commons license, and indicate if changes were made. The Creative Commons Public Domain Dedication waiver (http://creativecommons.org/publicdomain/zero/1.0/) applies to the data made available in this article, unless otherwise stated. 
cancer types [12-15]. However, the clinical significance of PD-L1 expression in NPC is controversial due to conflicting data amongst studies [16-19].

BRAF is one of downstream of EGFR pathway molecule [20], and BRAF (V600E) mutation is rarely reported in previous study [21]. In other solid tumors such as melanoma and non-small cell lung cancer, BRAF inhibitors were approved for patients with BRAF mutation positive.

In the present study we aim to evaluate the clinical significance of PD-L1, BARF and EGFR expressions in the tumor cells of a cohort of NPC patients. Separate data from this cohort of patients have been reported in a previous publication [7].

\section{Methods}

\section{Patient selection}

Consecutive patients who were pathologically diagnosed with NPC between 2006 and December 2010 at the Kiang Wu Hospital (Macau SAR of China) and for whom freshfrozen tissue samples were available were included. The clinicopathologic information of all patients was collected, including sex, age, tumor stage, pathologic type, and treatment methods and outcomes. Tumor stage was classified according to the International Union Against Cancer and American Joint Committee on Cancer staging system for NPC, seventh edition. Fresh nasopharyngeal tissue samples were obtained from all patients. The protocol was approved by the institutional review board of the Kiang Wu Hospital (KWH 2016-014).

\section{Treatment and outcome}

All patients received standard treatment including radiation therapy with or without chemotherapy. Briefly, the intensity modulated radiotherapy technique technology were utilized for radiation. Chemotherapy were given for patients based on their tumor stage and the decision by each patient's physician. Chemotherapy regimen was based on NCCN guidelines.

We defined progression-free survival (PFS) as time from date of treatment to the date of disease progression or death from any causes, whichever came first. Overall survival (OS) was defined as the time from date of treatment to the time of death.

\section{Immunohistochemistry for PD-L1, BRAF, and EGFR expression}

PD-L1, BRAF and EGFR expressions in the tumor cells was evaluated using immunohistochemistry. Four mm-thick sections were prepared from paraffin-embedded specimens of the NPC tumor. The sections were deparaffinized in xylene followed by $95 \%$ ethanol. After rehydration, sections were pretreated in a microwave oven at $95^{\circ} \mathrm{C}$ for $15 \mathrm{~min}$ in citrate buffer (pH 6.0) for antigen retrieval. Next, endogenous peroxidase activity was blocked with $4 \%$ Block ACE Powder in $\mathrm{H} 2 \mathrm{O}$ at $37^{\circ} \mathrm{C}$ for $10 \mathrm{~min}$.

\section{Immunohistochemistry (IHC) was carried out by benchmark XT automated stainer}

PD-L1 protein was detected by using PD-L1 (SP263) rabbit monoclonal antibody with Ultraview detection system (Ventana, Tucson, Arizona). Reference to the interpretation guide of Ventana PD-L1 (SP263) assay staining of non-small cell lung cancer, the tumor cells was counted if any intensity of the staining result demonstrating in membrane with a discontinuous, circumferential or basolateral pattern or rarely in peri-nuclear dot-like body.

BRAF V600E protein was detected by using BRAF V600E (VE1) mouse monoclonal primary antibody and the OptiView DAB IHC Detection Kit (Ventana, Tucson, Arizona). The immunostaining result was interpreted as positive if any intensity of cytoplasmic staining.

EGFR mutation specific antibodies were detected by using EGFR mutation specific rabbit monoclonal antibodies against del E746-A750 (6B6, dilution:1:50; Cell Signaling Technology, Inc., Boston, MA, USA) and L858R (43B2, dilution:1:10; Cell Signaling Technology, Inc). The immunoreactions were detected by OptiView DAB IHC Detection Kit (Ventana, Tucson, Arizona).

The immunostaining results were interpreted as positive if any intensity on cytoplasmic and/or membrane staining.

\section{Assessment of PD-L1, BRAF, and EGFR expression}

PD-L1, BRAF mutation, and EGFR mutation expression in tumor cells were evaluated in a blind fashion without knowledge of any existing clinical characteristics. Any staining within the tumor cell membrane or cytoplasm was considered positive. Grading was based on staining ratio of the tumor cells, $\geq 50 \%$ of tumor cells positive was scored as $3 ; \geq 5$ to $<50 \%(5-49 \%)$ of tumor cells expressed positive as 2 ; $\geq 1$ to $<5 \%(1-5 \%)$ of tumor cells expressed positive as 1 ; negative as 0 .

BRAF and EGFR mutation expression were categorized as negative or positive.

\section{Statistical analysis}

Fisher's exact test or the chi-squared test was performed to examine the association between PD-L1 expression and the oncogenic mutations versus various clinicopathological features, as appropriate. The PD-L1 expression was evaluated as a categorical variable $(0,1-5 \%, 5-49 \%$ and $\geq 50 \%$ expression). Survival curves were plotted using the Kaplan-Meier method and compared using a log-rank test. The prognostic impact of relevant clinicopathological variables including PD-L1 expression in the pulmonary metastatic tumors was evaluated using the Cox proportional hazards regression models and hazard 
ratios (HRs). To assess the prognostic value of high $\mathrm{PD}-$ L1 expression, variables with $P<0.2$ in the univariate analysis were entered into the multivariate analysis, and variables with $P<0.05$ were included in a final model with backward elimination methods. A two-sided $P$ value $<0.05$ was considered statistically significant. Statistical analyses were performed using the SPSS version 20.0 software package (SPSS Inc., Chicago, IL, USA).

\section{Results}

\section{Patient characteristics}

A total of 154 patients were included in the analysis. The baseline characteristics of patients are shown in Table 1. Median age was 60 years (range $26-83$ years). The majority of patients were male $(75.2 \%)$. All patients were diagnosed with non-keratinizing undifferentiated carcinoma according to the WHO histological classification. The median and maximum follow-up duration was 76 months and 145 months, respectively. The last day of follow-up was in January 2019. Seventy six patients (49.4\%) had tumor recurrence or metastasis. None of the patients received anti-PD-L1 antibody treatment because anti-PD-L1 antibody treatment was not available in Macau during the follow-up period.

\section{Expression of PD-L1, BRAF and EGFR}

Fig. 1

Relationship between PD-L1, BRAF mutation, and EGFR mutation expression with patient characteristics

PD-L1 expression was detected in $87.6 \%$ of biopsy tissue. PD-L1 expression was $0 \%$ in $11.0 \%$ of patients, $1-5 \%$ in $14.3 \%$ of patients, $5-49 \%$ in $47.4 \%$ of patients and $\geq 50 \%$ in $26 \%$ of patients. There was no difference in PD-L1 expression between genders or age groups. However, there was significantly higher expression of PD-L1 among patients with disease recurrence or metastasis $(P=0.001)$. There was also a significantly higher expression of BRAF mutation among patients with disease recurrence or metastasis $(P=0.035)$. There was no significant association between PD-L1 expression levels, BRAF V600E mutation and EGFR 19del mutation with age, sex or disease stage. Most of the tumor tissues that expressed PD-L1 were BRAF V600E mutation negative $(P=0.002)$. There was no significant association between PD-L1 expression levels and EGFR 19del mutation $(P=0.161)$.

\section{Prognostic impact on progression free survival and overall survival}

PD-L1 expression was significantly associated with overall survival. Higher expressions of PD-L1 were associated with shorter PFS $(P<0.001$, Fig. $2 \mathrm{a})$ and reduced OS ( $\mathrm{P}<0.001$, Fig. $2 \mathrm{~b})$. The 5-year PFS rates for patients with PD-L1 expression 0\%, 1-5\%, 5-49\% and $\geq 50 \%$
Table 1 Patient demographics and disease characteristics

\begin{tabular}{|c|c|c|c|}
\hline \multirow[t]{2}{*}{ Characteristic } & & \multicolumn{2}{|c|}{$N=154$} \\
\hline & & Cases & Percentage (\%) \\
\hline \multicolumn{4}{|l|}{ Age (Years) } \\
\hline & Median & 60 & \\
\hline & Range & $26-83$ & \\
\hline & $<60$ & 71 & 46.1 \\
\hline & $\geq 60$ & 83 & 53.9 \\
\hline \multicolumn{4}{|l|}{ Sex } \\
\hline & Male & 116 & 75.2 \\
\hline & Female & 38 & 24.8 \\
\hline \multicolumn{4}{|l|}{ Stage } \\
\hline & $|-| \mid$ & 67 & 43.5 \\
\hline & III-IV & 86 & 56.5 \\
\hline \multicolumn{4}{|l|}{ Treatment } \\
\hline & Chemoradiation & 124 & 80.5 \\
\hline & Radiation Only & 31 & 19.5 \\
\hline \multicolumn{4}{|l|}{ ECOG } \\
\hline & $0-1$ & 131 & 85.0 \\
\hline & $\geq 2$ & 24 & 15.0 \\
\hline \multicolumn{4}{|l|}{ Progression } \\
\hline & Yes & 76 & 49.4 \\
\hline & No & 78 & 50.6 \\
\hline \multicolumn{4}{|l|}{ PD-L1 } \\
\hline & $0 \%$ & 17 & 11.0 \\
\hline & $1-5 \%$ & 22 & 14.3 \\
\hline & $5-49 \%$ & 73 & 47.4 \\
\hline & $\geq 50$ & 40 & 26.0 \\
\hline & Unknown & 2 & 1.3 \\
\hline \multicolumn{4}{|l|}{ BRAF V600E } \\
\hline & Negative & 139 & 90.3 \\
\hline & Positive & 13 & 8.4 \\
\hline & Unknown & 2 & 1.3 \\
\hline \multicolumn{4}{|l|}{ EGFR 19del } \\
\hline & Negative & 149 & 96.8 \\
\hline & Positive & 3 & 1.9 \\
\hline & Unknown & 2 & 1.3 \\
\hline \multicolumn{4}{|l|}{ EGFR L858R } \\
\hline & Negative & 151 & 98.1 \\
\hline & Positive & 0 & 0.0 \\
\hline & Unknown & 3 & 1.9 \\
\hline
\end{tabular}

Values are presented as number (\%) unless otherwise stated

Percentages may not sum to exactly 100 due to rounding

were 75.5, 72.7, 55.9 and $24.8 \%$, respectively $(\mathrm{P}<0.001)$. The median PFS was 25 months (95\% CI 15.7-34.3 months) for patients with PD-L1 expression $\geq 50 \%$, and not yet reached for patients with PD-L1 expression < 


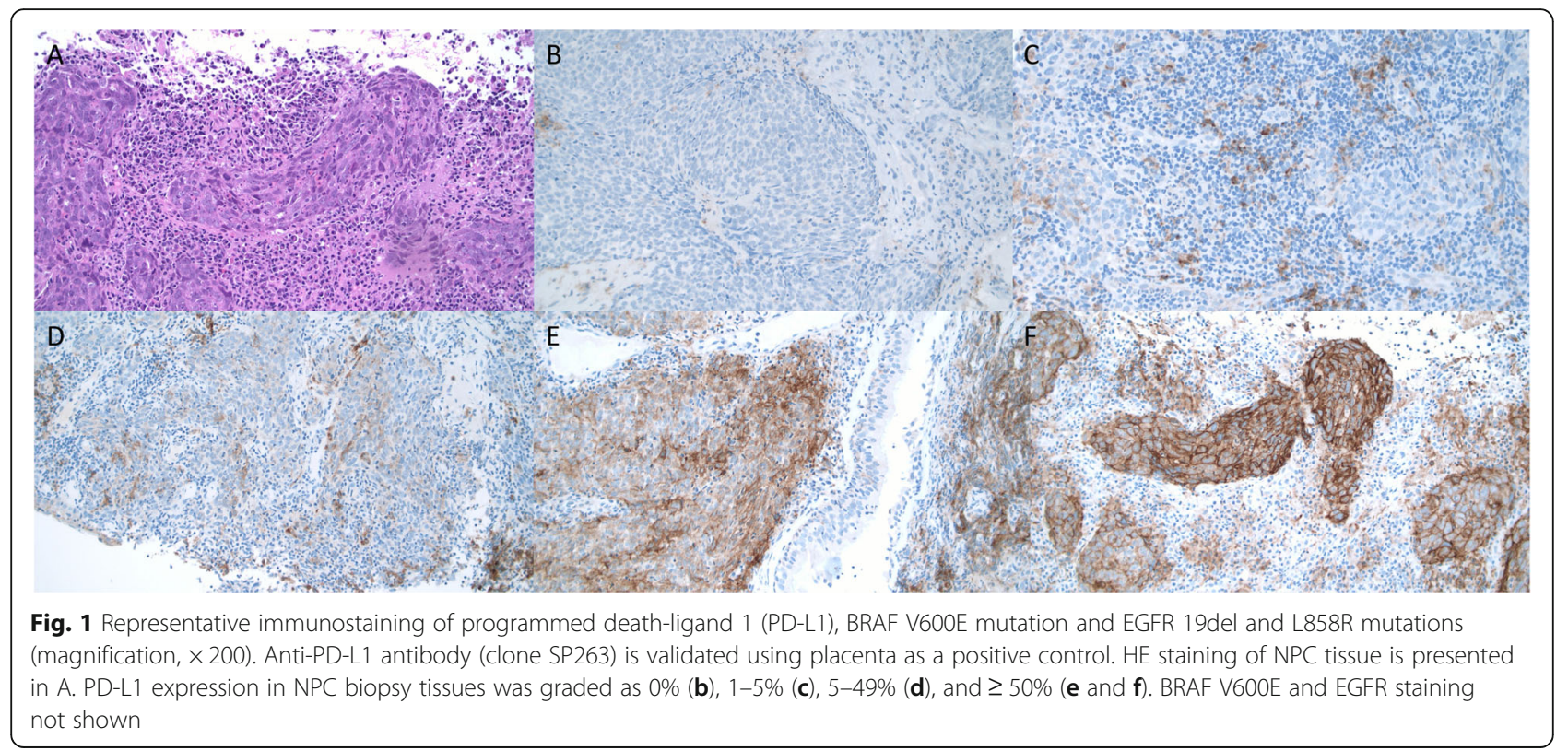

50\%. The overall median PFS for all patients was 84 months. The 5-year OS rate for patients with PD-L1 expression $0 \%, 1-5 \%, 5-49 \%$ and $\geq 50 \%$ were $85.7,72.7$, 68.3 and $35.0 \%$, respectively $(P<0.001)$. The median OS was 35 months (95\% CI 22.60-47.4 months) for patients with PD-L1 expression $\geq 50 \%$, and not yet reached for patients with PD-L1 expression $<50 \%$. The overall median OS for all patients was 96 months (95\% CI 60.2131.8 months). Consistent with our previous report, female patients had a favorable prognosis than male patients $(P=0.009$, figure not show).

PFS was significantly different between BRAF mutation negative and positive patients (5-year PFS: $55.1 \%$ vs. $30.8 \%, P=0.044$; Fig. 2c). However, OS rates did not differ significantly between BRAF mutation negative and positive patients (5-year OS rate: $61.9 \%$ vs. $46.2 \%, P=$ 0.075; Fig. 2d).

The results of prognostic factor analysis for survival using Cox proportional hazards regression model are shown in Fig. 3. Univariate analysis showed that high PDL1 expression and the female gender were significantly associated with a shorter OS. Multivariate analysis indicated that high PD-L1 expression, along with gender is associated with shorter OS and thus poorer prognosis. There was no interaction between PD-L1 expression and gender (Table 2). The presence of BRAF V600E mutation was associated with disease progression $(P=0.035$; Table 2$)$.

\section{Discussion}

It is known that PD-L1 expression is upregulated on various tumor cell lines. NPC is an EBV-associated cancer. Previous studies demonstrated that EBV-related latent membrane protein 1 (LMP1) and interferon-gamma
(IFN- $\gamma$ ) may upregulate PD-L1 in NPC $[22,23]$ and NK/ $\mathrm{T}$ cell lymphoma [24]. Expression of viral proteins, such as EBV nuclear antigen-1 or LMP1 and 2 in NPC cells can elicit a virus-specific immune response in patients with NPC. LMP1 expression and IFN- $\gamma$ activation can synergistically induce the expression of PD-L1 in NPC cells [22]. Expression of PD-L1 can also be upregulated by tumor-infiltrating lymphocytes (TILs), which is associated with impaired effector function (cytokine production and cytotoxic efficacy against tumor cells) and poor outcomes in NPC [25]. In our study, we found positive PD-L1 expression in $87.7 \%$ of patients with NPC; $14.3 \%$ had $0-1 \%$ PD-L1 expression, $47.4 \%$ had $1-49 \%$ expression while $26 \%$ had $\geq 50 \%$ expression. This is consistent with other studies which reported PD-L1 expression in $89-95 \%$ of NPC tumors, with $50 \%$ or more malignant cells being PDL1 positive in the majority of these tumors [26].

Activation of PD-1 pathway can lead to $\mathrm{T}$ cell exhaustion. Thus, the PD-1/PD-L1 axis is crucial in regulating anti-tumor immunity. In this study, we performed a retrospective analysis on 154 consecutive patients who were homogeneously treated with IMRT. Our findings demonstrate that high PD-L1 expression is a poor prognostic factor for NPC patients. Best progression free survival was seen in the PD-L1 expression negative group, with a 5year PFS rate of $75.2 \%$. For patients with positive PD-L1 expression, the PFS rate reduces as expression levels increase; 5-year PFS rates were 72.7, 55.9 and $24.8 \%$ for patients with PD-L1 expression $1-5 \%, 5-49 \%$ and $\geq 50 \%$, respectively. The 5-year OS rate for patients with PD-L1 expression $0 \%, 1-5 \%, 5-49 \%$ and $\geq 50 \%$ were $85.7,72.7$, 68.3 and $35.0 \%$, respectively. Our data are consistent to those recently published by Ben-Betzalel et al. [19, 27-29] 


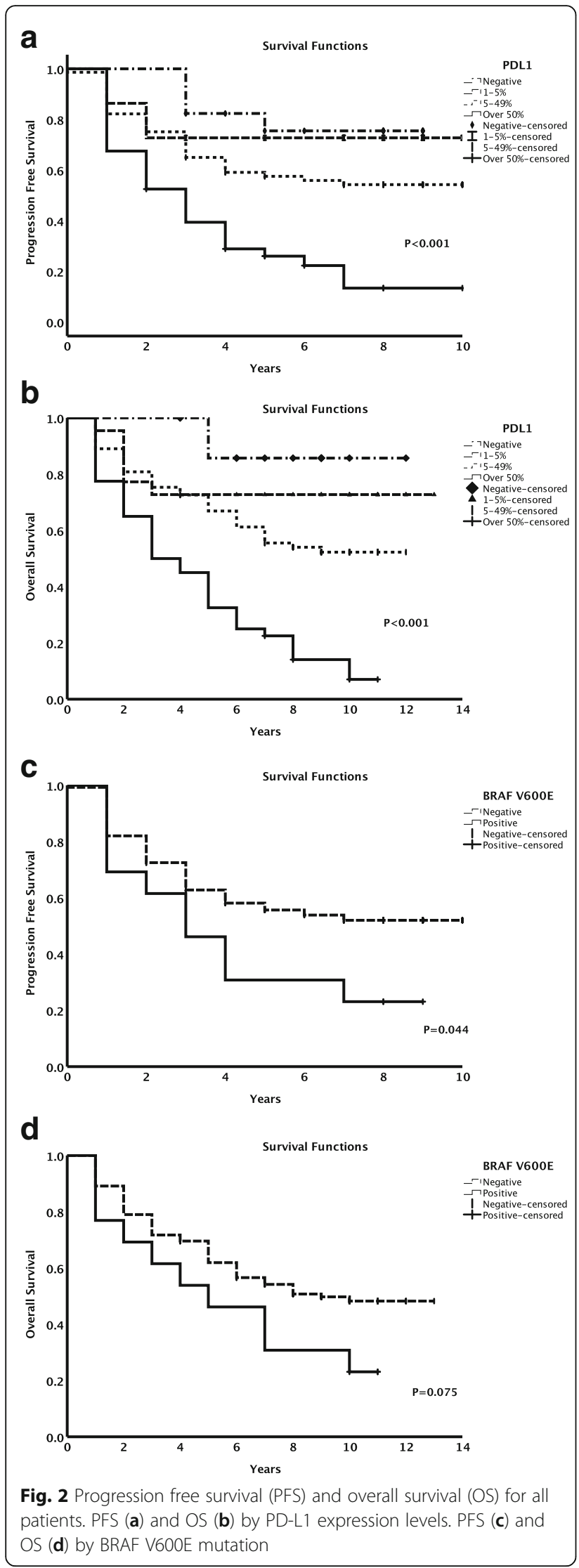

who found similar association of PD-L1 expression with poor survival. However, other studies have reported favorable prognosis with increased PD-L1 expression [17, 30], while others found no relation between PD-L1 expression and survival [31,32].

There many reasons behind these inconsistent findings. First, some studies included a mixed patient population, which consists of patients with NPC patients as well as those with other types of head and neck squamous cancer. Second, not all studies used commercially available clones of PD-L1 antibodies. SP263 and 22C3 (Dako), and SP142 (Ventana) have been shown to pass the Western Blot and immunohistochemical validation. In prior comparison trials, it was shown that $22 \mathrm{C} 3$ and SP263 were closely aligned in tumor cell staining, but SP142 stains less tumor cells [33]. Third, the follow up period of some of the studies were too short for PFS and OS analysis. Our study focused on NPC patients and with an extended follow-up period of 13 years. Since the percentage of PD-L1-positive cells can vary due to different antibody clones and immunostaining methods, finding the best cutoff value with the highest clinical significance is crucial in such studies. We used the SP263 antibody with the standard cut off value of 1 and $5 \%$, which is frequently used for lung cancer and other cancer types [34]. Inevitably, whenever an IHC-based biomarker is considered, questions will arise regarding the reproducibility of the staining of the tissue and consistency in interpretation of the test by pathologists. In future, multicenter, international standardization efforts could address many of these questions and help develop one "standardized" assay to analyse additional immunotherapy-related predictive markers [35].

BRAF mutations have been identified in melanoma and colorectal cancer, but is rarely reported in NPC [36]. BRAF mutations are associated with poorer survival in patients with melanoma [37], but the significance of BRAF mutations among NPC patients has not been thoroughly investigated. For the first time, we report that the BRAF V600E mutation was significantly associated with disease progression and PFS. In this study, 13 of 154 patients (8.4\%) were BRAF V600E mutation positive. The 5-year PFS of BRAF V600E mutation positive and negative patients were 55.1 and $30.8 \%$, respectively.

Using multivariate analysis, PD-L1 expression and gender were independent prognostic factors for overall survival. This confirmed our previous study, that female patients had a favorable prognosis than male patients.

PD-L1 expression is the most extensively studied biomarker with respect to predicting the efficacy of anti-PD1 or anti-PD-L1 therapies. A positive correlation between PD-L1 expression and treatment efficacy has been reported in the study of nivolumab [38] and pembrolizumab for NPC [39]. In our center, 70 patients with NPC have 


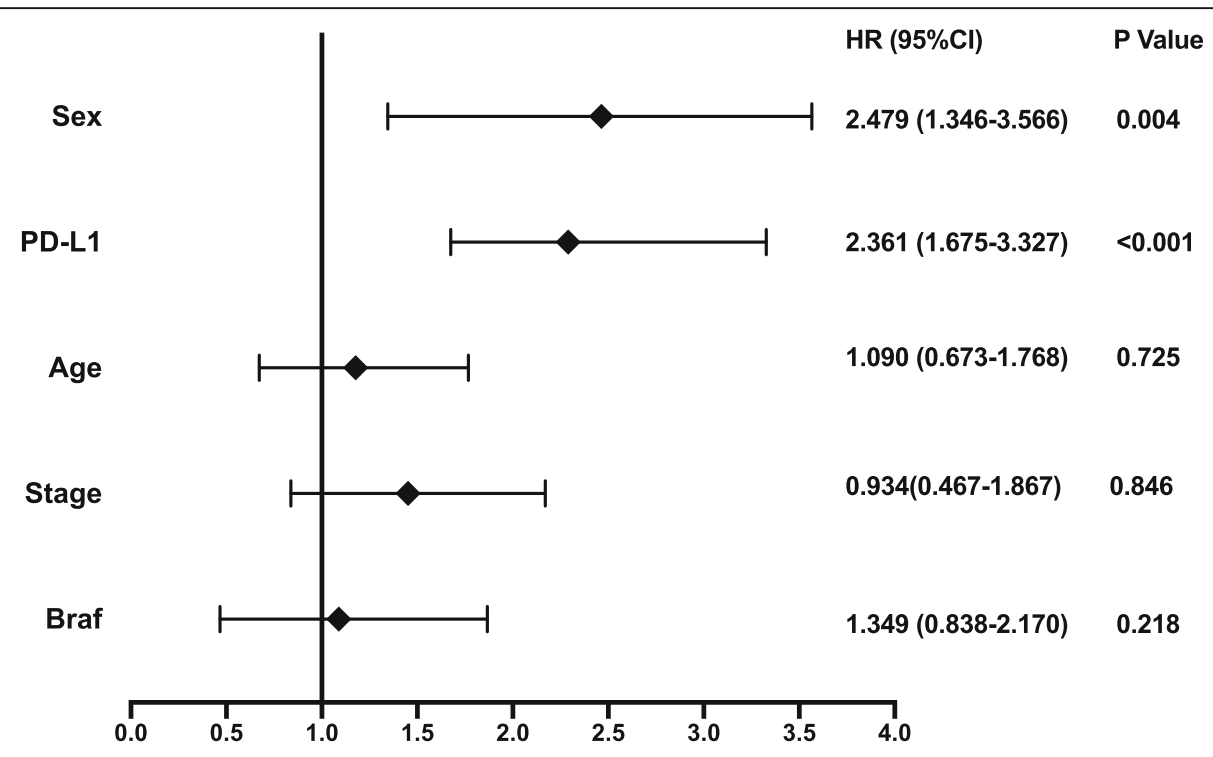

Fig. 3 Forest plot of hazard ratio (HR) for overall survival (OS) by independent prognostic factors

received PD-1 therapy, 13 received nivolumab monotherapy, 29 received pembrolizumab monotherapy and 28 received pembrolizumab combined with chemotherapy. An internal analysis of these patients revealed that PD-L1 positive tumor cell with high CD8 positive tumor infiltrates correlated with objective response to PD-L1 inhibitor (data not published).

Our study has some limitations. Our study lacks EBV loading data as the EBV DNA test was not routinely carried out during the period that patients received treatment. EBV expression is an import contributor here and may increase PD11 expression [24, 40]. Secondly, patients in this study did not receive PD-1 or PD-L1 targeted therapy as these were not available in Macau during the follow-up period. Therefore, we were unable to explore the correlation between PDL1 expression and the efficacy of immunotherapy.

\section{Conclusion}

Our results suggest that high tumor PD-L1 expression and BRAF V600E mutation are associated with poor outcomes in patients with NPC. PD-L1 expression was found to be a significant prognostic factor, and high PD-L1 expression may be of prognostic value for disease progression and survival.

Table 2 Association between clinical parameters and expression of PD-L1, BRAF and EGFR proteins

\begin{tabular}{|c|c|c|c|c|c|c|c|c|c|c|c|}
\hline \multirow[t]{2}{*}{ Characteristic } & \multicolumn{5}{|c|}{ PD-L1 (N) } & \multicolumn{3}{|c|}{ BRAF V600E (N) } & \multicolumn{3}{|c|}{ EGFR 19Del (N) } \\
\hline & 0 & $1-5$ & $5-49$ & $>50$ & P-value & Negative & Positive & P-value & Negative & Positive & P-value \\
\hline \multicolumn{12}{|l|}{ Age } \\
\hline$<60$ & 10 & 11 & 36 & 12 & 0.127 & 64 & 5 & $0.411^{*}$ & 67 & 2 & $0.431^{*}$ \\
\hline$\geq 60$ & 7 & 11 & 37 & 28 & & 75 & 8 & & 82 & 1 & \\
\hline \multicolumn{12}{|l|}{ Sex } \\
\hline Male & 14 & 17 & 53 & 30 & 0.776 & 104 & 10 & $0.602^{*}$ & 112 & 2 & $0.581^{*}$ \\
\hline Female & 3 & 5 & 20 & 10 & & 35 & 3 & & 37 & 1 & \\
\hline \multicolumn{12}{|l|}{ Stage } \\
\hline$|-| \mid$ & 7 & 9 & 36 & 14 & 0.517 & 60 & 6 & $0.529^{*}$ & 65 & 1 & $0.599^{*}$ \\
\hline III-IV & 10 & 13 & 37 & 26 & & 79 & 7 & & 84 & 2 & \\
\hline \multicolumn{12}{|l|}{ Progression } \\
\hline Yes & 4 & 6 & 32 & 33 & 0.001 & 65 & 10 & $0.035^{*}$ & 73 & 2 & $0.490^{*}$ \\
\hline No & 13 & 16 & 41 & 7 & & 74 & 3 & & 76 & 1 & \\
\hline
\end{tabular}

*: Fisher's exact test

Chi-squared test was used in variables without * 


\section{Abbreviations}

PD-L1: programmed death-ligand 1 (PD-L1); NPC: nasopharyngeal carcinoma (NPC); EGFR: epidermal growth factor receptor; PFS: progression-free survival; OS: overall survival; IHC: Immunohistochemistry; LMP-1: latent membrane protein 1; EBV: Epstein-Barr virus

\section{Acknowledgements}

None.

\section{Authors' contributions}

YBC and TYL conceived of the presented idea. YBC and JIC carried out the experiment. GLX, YQC, XBQ, SCM and $\mathrm{HH}$ collected data. YBC verified the analytical methods. Both YBC and TYL contributed to the final version of the manuscript. All authors have read and approved the manuscript.

\section{Funding}

This study is funded by The Science and Technology Development Fund (FDCT) of Macau, grant number is 019/2016/AFJ. This funding source had no role in study design, data collection and analysis, decision to publish, or preparation of the manuscript.

\section{Availability of data and materials}

The datasets used and analyzed during the current study are available from the corresponding author on reasonable request.

\section{Ethics approval and consent to participate}

The protocol was approved by the institutional review board of the Kiang Wu Hospital (KWH 2016-014).

Declarations: The need for consent to participate was waived by the institutional review board of the Kiang Wu Hospital on June 21, 2019

\section{Consent for publication}

No details, images, or videos relating to an individual person was included in this paper therefore written informed consent for the publication of these details was not obtained from any person.

\section{Competing interests}

The authors declare that they have no competing interests.

\section{Author details}

'Department of Oncology, Kiang Wu Hospital, Macau, SAR, China 2Department of Pathology, Kiang Wu Hospital, Macau, SAR, China. ${ }^{3}$ Department of Oncology, Sun Yat-Sen University Cancer Center, Guangzhou, China.

\section{Received: 25 June 2019 Accepted: 18 October 2019}

\section{0 (a)}

\section{References}

1. Chen W, Zheng R, Baade PD, Zhang S, Zeng H, Bray F, et al. Cancer statistics in China, 2015. CA Cancer J Clin. 2016;66(2):115-32.

2. Chen W, Xia C, Zheng R, Zhou M, Lin C, Zeng H, et al. Disparities by province, age, and sex in site-specific cancer burden attributable to 23 potentially modifiable risk factors in China: a comparative risk assessment. Lancet Glob Health. 2019:7(2):e257-e69.

3. Blanchard P, Lee A, Marguet S, Leclercq J, Ng WT, Ma J, et al. Chemotherapy and radiotherapy in nasopharyngeal carcinoma: an update of the MAC-NPC meta-analysis. Lancet Oncol. 2015;16(6):645-55.

4. Yang L, Xia L, Wang Y, He S, Chen H, Liang S, et al. Development and external validation of nomograms to predict the risk of skeletal metastasis at the time of diagnosis and skeletal metastasis-free survival in nasopharyngeal carcinoma. BMC Cancer. 2017;17(1):628.

5. Lee AW, Ma BB, Ng WT, Chan AT. Management of Nasopharyngeal Carcinoma: current practice and future perspective. J Clin Oncol. 2015; 33(29):3356-64.

6. Zhang L, Huang Y, Hong S, Yang Y, Yu G, Jia J, et al. Gemcitabine plus cisplatin versus fluorouracil plus cisplatin in recurrent or metastatic nasopharyngeal carcinoma: a multicentre, randomised, open-label, phase 3 trial. Lancet. 2016;388(10054):1883-92.

7. Xiao G, Cao Y, Qiu X, Wang W, Wang Y. Influence of gender and age on the survival of patients with nasopharyngeal carcinoma. BMC Cancer. 2013;13:226.
8. Tang XR, Li YQ, Liang SB, Jiang W, Liu F, Ge WX, et al. Development and validation of a gene expression-based signature to predict distant metastasis in locoregionally advanced nasopharyngeal carcinoma: a retrospective, multicentre, cohort study. Lancet Oncol. 2018:19(3):382-93.

9. Ono T, Azuma K, Kawahara A, Sasada T, Matsuo N, Kakuma T, et al. Prognostic stratification of patients with nasopharyngeal carcinoma based on tumor immune microenvironment. Head Neck. 2018;40(9):2007-19.

10. Cho YA, Yoon HJ, Lee Jl, Hong SP, Hong SD. Relationship between the expressions of PD-L1 and tumor-infiltrating lymphocytes in oral squamous cell carcinoma. Oral Oncol. 2011;47(12):1148-53.

11. Mattox AK, Lee J, Westra WH, Pierce RH, Ghossein R, Faquin WC, et al. PD-1 expression in head and neck squamous cell carcinomas derives primarily from functionally Anergic CD4(+) TlLs in the presence of PD-L1(+) TAMs. Cancer Res. 2017;77(22):6365-74.

12. Dang TO, Ogunniyi A, Barbee MS, Drilon A. Pembrolizumab for the treatment of PD-L1 positive advanced or metastatic non-small cell lung cancer. Expert Rev Anticancer Ther. 2016;16(1):13-20.

13. Doroshow DB, Sanmamed MF, Hastings K, Politi K, Rimm DL, Chen L, et al. Immunotherapy in non-small cell lung Cancer: facts and hopes. Clin Cancer Res. 2019.

14. Koemans WJ, Chalabi M, van Sandick JW, van Dieren JM, Kodach LL. Beyond the PD-L1 horizon: in search for a good biomarker to predict success of immunotherapy in gastric and esophageal adenocarcinoma. Cancer Lett. 2019;442:279-86.

15. Taube JM, Klein A, Brahmer JR, Xu H, Pan X, Kim JH, et al. Association of PD1, PD-1 ligands, and other features of the tumor immune microenvironment with response to anti-PD-1 therapy. Clin Cancer Res. 2014;20(19):5064-74

16. Li YF, Ding JW, Liao LM, Zhang ZL, Liao SS, Wu Y, et al. Expression of programmed death ligand-1 predicts poor outcome in nasopharyngeal carcinoma. Mol Clin Oncol. 2017;7(3):378-82.

17. Liu YJ, Tsang NM, Hsueh C, Yeh CJ, Ueng SH, Wang TH, et al. Low PD-L1 Expression Strongly Correlates with Local Recurrence in Epstein-Barr VirusPositive Nasopharyngeal Carcinoma after Radiation-Based Therapy. Cancers (Basel). 2018;10(10).

18. Yang WF, Wong MCM, Thomson PJ, Li KY, Su YX. The prognostic role of PDL1 expression for survival in head and neck squamous cell carcinoma: a systematic review and meta-analysis. Oral Oncol. 2018;86:81-90.

19. Zhou Y, Shi D, Miao J, Wu H, Chen J, Zhou X, et al. PD-L1 predicts poor prognosis for nasopharyngeal carcinoma irrespective of PD-1 and EBV-DNA load. Sci Rep. 2017;7:43627.

20. Fernandez-Medarde A, Santos E. Ras in cancer and developmental diseases. Genes Cancer. 2011;2(3):344-58.

21. Zhang JW, Qin T, Hong SD, Zhang J, Fang WF, Zhao YY, et al. Multiple oncogenic mutations related to targeted therapy in nasopharyngeal carcinoma. Chin J Cancer. 2015;34(4):177-83.

22. Fang W, Zhang J, Hong S, Zhan J, Chen N, Qin T, et al. EBV-driven LMP1 and IFN-gamma up-regulate PD-L1 in nasopharyngeal carcinoma: implications for oncotargeted therapy. Oncotarget. 2014:5(23):12189-202.

23. Yoshizaki T, Kondo S, Endo K, Nakanishi Y, Aga M, Kobayashi E, et al. Modulation of the tumor microenvironment by Epstein-Barr virus latent membrane protein 1 in nasophanyngeal carcinoma. Cancer Sci. 2018;109(2):272-8.

24. Bi XW, Wang H, Zhang WW, Wang JH, Liu WJ, Xia ZJ, et al. PD-L1 is upregulated by EBV-driven LMP1 through NF-kappaB pathway and correlates with poor prognosis in natural killer/T-cell lymphoma. J Hematol Oncol. 2016;9(1):109.

25. Hsu MC, Hsiao JR, Chang KC, Wu YH, Su IJ, Jin YT, et al. Increase of programmed death-1-expressing intratumoral CD8 T cells predicts a poor prognosis for nasopharyngeal carcinoma. Mod Pathol. 2010;23(10):1393-403.

26. Chen BJ, Chapuy B, Ouyang J, Sun HH, Roemer MG, Xu ML, et al. PD-L1 expression is characteristic of a subset of aggressive B-cell lymphomas and virus-associated malignancies. Clin Cancer Res. 2013;19(13):3462-73.

27. Zhao L, Liao X, Hong G, Zhuang Y, Fu K, Chen P, et al. Mismatch repair status and high expression of PD-L1 in nasopharyngeal carcinoma. Cancer Manag Res. 2019;11:1631-40

28. Zhang J, Fang W, Qin T, Yang Y, Hong S, Liang W, et al. Co-expression of PD-1 and PD-L1 predicts poor outcome in nasopharyngeal carcinoma. Med Oncol, 2015:32(3):86.

29. Qu Y, Wang D, Yang L, Liu HY, Cui W, Che YQ. Expression and clinical significance of programmed death ligand 1 in nasopharyngeal carcinoma. Mol Clin Oncol. 2018;9(1):75-81. 
30. Lee VH, Lo AW, Leung CY, Shek WH, Kwong DL, Lam KO, et al. Correlation of PD-L1 expression of tumor cells with survival outcomes after radical intensity-modulated radiation therapy for non-metastatic nasopharyngeal carcinoma. PLoS One. 2016;11(6):e0157969.

31. Chang AMV, Chiosea SI, Altman A, Pagdanganan HA, Ma C. Programmed death-ligand 1 expression, microsatellite instability, Epstein-Barr virus, and human papillomavirus in nasopharyngeal carcinomas of patients from the Philippines. Head Neck Pathol. 2017;11(2):203-11.

32. Larbcharoensub N, Mahaprom K, Jiarpinitnun C, Trachu N, Tubthong N, Pattaranutaporn P, et al. Characterization of PD-L1 and PD-1 expression and CD8+ tumor-infiltrating lymphocyte in Epstein-Barr virus-associated nasopharyngeal carcinoma. Am J Clin Oncol. 2018;41(12):1204-10.

33. Lantuejoul S, Damotte D, Hofman V, Adam J. Programmed death ligand 1 immunohistochemistry in non-small cell lung carcinoma. J Thorac Dis. 2019; 11(Suppl 1):S89-S101.

34. Marchetti A, Barberis M, Franco R, De Luca G, Pace MV, Staibano S, et al. Multicenter comparison of 22C3 PharmDx (Agilent) and SP263 (Ventana) assays to test PD-L1 expression for NSCLC patients to be treated with immune checkpoint inhibitors. J Thorac Oncol. 2017;12(11):1654-63.

35. Kerr KM, Tsao MS, Nicholson AG, Yatabe Y, Wistuba II, Hirsch FR, et al. Programmed death-ligand 1 immunohistochemistry in lung Cancer: in what state is this art? J Thorac Oncol. 2015;10(7):985-9.

36. Chou CC, Chou MJ, Tzen CY. PIK3CA mutation occurs in nasopharyngeal carcinoma but does not significantly influence the disease-specific survival. Med Oncol. 2009;26(3):322-6.

37. Houben R, Becker JC, Kappel A, Terheyden P, Brocker EB, Goetz R, et al. Constitutive activation of the Ras-Raf signaling pathway in metastatic melanoma is associated with poor prognosis. J Carcinog. 2004;3:6.

38. Ma BBY, Lim WT, Goh BC, Hui EP, Lo KW, Pettinger A, et al. Antitumor activity of Nivolumab in recurrent and metastatic nasopharyngeal carcinoma: an international, multicenter study of the Mayo Clinic phase 2 consortium (NCl-9742). J Clin Oncol. 2018;36(14):1412-8.

39. Hsu C, Lee SH, Ejadi S, Even C, Cohen RB, Le Tourneau C, et al. Safety and antitumor activity of Pembrolizumab in patients with programmed deathligand 1-positive nasopharyngeal carcinoma: results of the KEYNOTE-028 study. J Clin Oncol. 2017;35(36):4050-6.

40. Anastasiadou E, Stroopinsky D, Alimperti S, Jiao AL, Pyzer AR, Cippitelli C, et al. Epstein-Barr virus-encoded EBNA2 alters immune checkpoint PD-L1 expression by downregulating miR-34a in B-cell lymphomas. Leukemia. 2019;33(1):132-47.

\section{Publisher's Note}

Springer Nature remains neutral with regard to jurisdictional claims in published maps and institutional affiliations.

Ready to submit your research? Choose BMC and benefit from:

- fast, convenient online submission

- thorough peer review by experienced researchers in your field

- rapid publication on acceptance

- support for research data, including large and complex data types

- gold Open Access which fosters wider collaboration and increased citations

- maximum visibility for your research: over $100 \mathrm{M}$ website views per year

At $\mathrm{BMC}$, research is always in progress.

Learn more biomedcentral.com/submissions 